\title{
Relações alométricas da comunidade arbórea de diferentes áreas de uma floresta ombrófila mista do sul do Brasil
}

\section{Allometric relationships of the arboreal community in different areas of a mixed ombrophylous forest in south Brazil}

\author{
Ana Paula Liboni ${ }^{1}$; Diego Resende Rodrigues ${ }^{1}$; Bianca Buck Perina ${ }^{1}$; Vanessa \\ Patrícia Pereira Rosa ${ }^{1}$; Yves Rafael Bovolenta ${ }^{1}$; Edmilson Bianchini² ${ }^{2}$ José Antonio \\ Pimenta $^{2}$
}

Resumo

O estudo da alometria é importante para a compreensão de aspectos ecológicos e evolutivos em espécies de plantas, além de auxiliar no entendimento da estrutura e dinâmica das florestas. O objetivo deste estudo foi analisar as relações alométricas da comunidade arbórea de duas áreas em diferentes estádios sucessionais (mais avançado - área 1, e menos avançado - área 2) da Floresta Ombrófila Mista do Parque Ecológico da Klabin, Telêmaco Borba/PR/Brasil ( $\left.24^{\circ} 17^{\prime} \mathrm{S} 50^{\circ} 35^{\prime} \mathrm{W}\right)$. A comunidade arbórea da borda do fragmento em estádio sucessional mais avançado também foi incluída nas análises. Foi estabelecida uma transecção de $4 \mathrm{~m}$ de largura em cada área, onde foram amostrados 150 indivíduos arbóreos com altura igual ou maior que 1,5 m. Os indivíduos tiveram o diâmetro à altura do peito (DAP), a altura total e a altura do fuste mensurados. Foram realizadas as seguintes relações alométricas: altura total x DAP, altura total $\mathrm{x}$ altura de fuste e altura de fuste x DAP. Foi utilizada a análise de covariância para testar a diferença entre as retas, e teste a posteriori de Scheffé. As plantas das áreas 1 e 2 investiram mais em crescimento em diâmetro em relação à altura do que as plantas da borda, além disso, aquelas plantas da área em estádio sucessional mais avançado, em relação à altura do fuste, também investiram mais em diâmetro. A colonização da borda e da área de estádio sucessional menos avançado preferencialmente por espécies pioneiras, e as espécies de estádios mais avançados da sucessão que colonizam o subosque da área 1 podem ser as principais causas das diferenças na arquitetura dos indivíduos da comunidade arbórea das áreas estudadas.

Palavras-chave: Alometria. Floresta ombrófila mista. Comunidade arbórea.

\begin{abstract}
The study of allometry is important for understanding ecological and evolutionary aspects of plant species, and also in understanding the structure and dynamics of forests. This study aimed to assess the tree community allometric relationships of two areas in different successional stage (more advanced area 1, and less advanced - area 2) of a Mixed Ombrophylous Forest located in the Klabin's Ecological Park, Telêmaco Borba/Paraná state $\left(24^{\circ} 17^{\prime} \mathrm{S} 50^{\circ} 35^{\prime} \mathrm{W}\right)$. The edge arboreal community was also included in the analysis. A $4 \mathrm{~m}$ width transect was established in each area, where 150 arboreal individuals with height equal to or greater than 1,5 m were sampled. Individuals had the diameter at breast height (DBH), total height and branching height recorded. The allometric relationships studied were total height $\mathrm{x}$ $\mathrm{DBH}$, total height $\mathrm{x}$ branching height and branching height $\mathrm{x}$ DBH. Analysis of covariance was used to test allometric differences, and a posteriori Scheffé test. Plants of areas 1 and 2 invested more in diameter growth in relation to height, and also invested more in diameter growth in relation to branching height. Colonization of the edge and area 2 by pioneer species and the presence of understory species in area 1 may be the main causes of differences in the community tree architecture of the studied areas. Key words: Allometry. Mixed ombrophylous forest. Arboreal community.
\end{abstract}

\footnotetext{
1 Programa de Pós Graduação em Ciências Biológicas - Universidade Estadual de Londrina. E-mail: pimenta@uel.br

2 Docentes do Departamento de Biologia Animal e Vegetal - CCB - Universidade Estadual de Londrina.
} 


\section{Introdução}

A forma de crescimento das árvores tem grande importância ecológica (ARCHIBALD; BOND, 2003), pois afeta a interceptação da luz, o crescimento em altura, a defesa e a reprodução, determinando, portanto, a história de vida das espécies arbóreas (ARCHIBALD; BOND, 2003; KOHYAMA; HOTTA, 1990).

À medida que crescem, as plantas devem acumular uma quantidade crescente de biomassa para sustentação, em razão das pressões ambientais às quais são submetidas devido a fatores como a gravidade e o vento (WALLER, 1986). Por outro lado, devem investir em área fotossintética para manterem a assimilação de biomassa (O'BRIEN et al., 1995). O balanço entre esses investimentos reflete estratégias adaptativas das plantas e pode ser evidenciado através do estudo da relação entre tamanho e forma em plantas, denominado alometria (NIKLAS, 1994).

O estudo da alometria é um ponto importante para a compreensão de aspectos ecológicos e evolutivos em espécies de plantas e os modelos gerados a partir desses estudos podem ser ferramentas poderosas de previsão em ecologia (BOND; HONIG; MAZE, 1999), além de auxiliar no entendimento da estrutura e dinâmica das florestas (KING, 1996; SPOSITO; SANTOS, 2001)

Muitos caracteres morfológicos de um indivíduo variam dependendo da arquitetura específica, do microambiente e do grau de restrição ecológica onde se iniciou seu crescimento (KING, 1996; KOHYAMA, 1987; PORTELA; SANTOS, 2003). As relações alométricas também podem variar com a fase de desenvolvimento em que a planta se encontra, pois os indivíduos sofrem transformações mecânicas ao longo do seu crescimento (ALVES; SANTOS, 2002; NIKLAS, 1994). Portanto, a arquitetura de uma árvore adulta não reflete somente as condições às quais o indivíduo está submetido no presente, mas é o resultado dos fatores genéticos e ambientais que atuaram no seu crescimento desde o estádio de plântula (ARCHIBALD; BOND, 2003).

O processo de fragmentação de habitat é reconhecido atualmente como uma das principais ameaças à diversidade causando alterações em parâmetros populacionais (genéticos, estruturais e espaciais), podendo levar à extinção de espécies (SILVA et al., 2007). A fragmentação florestal altera variáveis ambientais importantes para as características adaptativas vegetais, tais como disponibilidade luminosa e hídrica (KAPOS; GANADE; MATSUI, 1993; KAPOS et al., 1997), o que influencia as interações biológicas como a competição, e, em última instância, pode ter efeitos sobre a densidade e as relações alométricas das plantas (BERTANI, 2006).

Ao longo da sucessão florestal secundária, mudanças na estrutura (área basal, densidade, estratificação do dossel), riqueza e composição de espécies, bem como em processos como ciclagem de nutrientes e produtividade primária, ocorrem através de uma sequência de eventos (GUARIGUATA; OSTERTAG，2001) e são dirigidas pelo tipo de alteração nas condições físicas e químicas do solo devido ao histórico de perturbação e uso da terra, e pela disponibilidade e disposição espacial das manchas de floresta remanescentes que funcionam como fontes de sementes para a regeneração (ALVES; METZGER, 2006; GUARIGUATA; OSTERTAG, 2001). Desta foram, o histórico de perturbações tem muitas implicações na sucessão florestal, pois diferentes tipos e intensidades de perturbações podem resultar em diferentes estruturas florestais (ALVES; METZGER, 2006; GUARIGUATA; OSTERTAG, 2001).

O objetivo deste trabalho foi avaliar a forma dos indivíduos da comunidade arbórea de uma área em estádio sucessional mais avançado e de uma área em estádio sucessional menos avançado da Floresta Ombrófila Mista do Parque Ecológico 
da Klabin, Telêmaco Borba/PR/Brasil, a fim de responder a seguinte questão: existem diferenças na arquitetura entre os indivíduos arbóreos das diferentes áreas?

\section{Material e métodos}

\section{Área de estudo}

O estudo foi realizado no Parque Ecológico da Klabin S.A., localizado no estado do Paraná, Brasil, nas coordenadas $24^{\circ} 17^{\prime} \mathrm{S} 50^{\circ} 35^{\prime} \mathrm{W}$. O parque é uma área particular com 11.196 ha sendo que deste total, segundo Fávaro et al. (2006) e Barbosa et al. (2007), 7.883 ha são ocupados por blocos dispersos de remanescentes de floresta nativa, interligados por diversos cursos d'água e florestas ciliares. O restante é ocupado por reflorestamentos de Araucaria angustifolia (Bert.) O. Kuntze, Eucalyptus spp. e Pinus spp.

$\mathrm{Na}$ paisagem local observa-se um mosaico formado por três tipos de formações vegetacionais nativas, a Floresta Estacional Semidecidual, a Floresta Ombrófila Mista e pequenas manchas de Campos Naturais (AZEVEDO et al., 2008).

O clima da região é classificado, segundo Köppen, como uma transição entre Cfa e $\mathrm{Cfb}$, sendo descrito como subtropical úmido com verões quentes a moderadamente quentes e invernos úmidos e frios, com média anual de precipitação de 1.700 mm e média anual de temperatura de 19,5 ${ }^{\circ} \mathrm{C}$ (MENDONÇA; DANNI-OLIVEIRA, 2002).

As áreas estudadas foram: (área 1) fragmento de Floresta Ombrófila Mista secundária em avançado estádio sucessional e bem conservada, sem histórico conhecido de perturbação, caracterizada por excepcionais exemplares de A. angustifolia (pinheiro-do-paraná), Patagonula americana L. (guajavira) e Eugenia involucrata DC. (cerejeira), com sub-bosque dominado por samambaias, bromélias, cipós e avencas; (área 2) fragmento de Floresta Ombrófila Mista secundária cujo histórico corresponde a uma pastagem que foi abandonada para regeneração natural a cerca de 50 anos.

Considerando que a criação de bordas florestais pode causar mudanças na abundância relativa e na composição de espécies de planta (NASCIMENTO; LAURANCE, 2006), em grande parte devido ao aumento no recrutamento e densidade de espécies arbóreas pioneiras (LAURANCE et al., 1998; WILLIAMS-LINERA, 1990) e diminuição na densidade de plântulas de espécies tardias (BENÍTEZ-MALVIDO, 1998), a borda do fragmento em estádio sucessional mais avançado foi incluída nas análises. Espera-se que a arquitetura dos indivíduos arbóreos da borda seja mais semelhante à dos indivíduos da área 2, que se apresenta em estádio sucessional menos avançado.

As áreas 1 e 2 estão separadas por uma distância de aproximadamente $2 \mathrm{~km}$ e ambas localizam-se em terreno plano. É importante ressaltar que a borda 1 está localizada no mesmo fragmento que a área 1, porém essas não são áreas consecutivas, estando separadas por cerca de $1 \mathrm{~km}$ de distância.

\section{Coleta e análise de dados}

Para o estudo das relações alométricas, foi estabelecida uma transecção de $4 \mathrm{~m}$ de largura em cada área, onde, independente da espécie, foram amostrados todos os indivíduos arbóreos com altura total igual ou maior que 1,5 m que apareceram até atingir 150 indivíduos em cada área. As transecções foram estabelecidas paralelamente à estrada que circunda os fragmentos e estavam distantes aproximadamente $100 \mathrm{~m}$ da estrada nas áreas 1 e 2 , e a $10 \mathrm{~m}$ da estrada na borda. O perímetro à altura do peito (PAP) de todos os indivíduos foi medido com fita métrica a uma altura de $1,30 \mathrm{~m}$ do solo, e posteriormente esse valor foi convertido para diâmetro à altura do peito (DAP) através da fórmula $\mathrm{D}=\mathrm{C} / \pi$. A altura total e altura do fuste foram estimadas visualmente, utilizando-se uma referência de comprimento conhecido, quando não foi possível medir com a fita métrica. 
As relações alométricas dos indivíduos são geralmente expressas por funções derivadas de regressões lineares das variáveis transformadas em logaritmos de base 10. A equação que expressa estas relações é: $\mathrm{y}=\mathrm{ax}{ }^{\mathrm{b}}$, ou $\log \mathrm{y}=\log \mathrm{a}+\mathrm{b} \log \mathrm{x}$, onde $\mathrm{a}$ e b são parâmetros obtidos através de regressão linear (KOHYAMA; HOTTA, 1990; SOKAL; ROHLF, 1981). Nas comparações da forma dos indivíduos entre as áreas, as diferenças poderão ocorrer tanto em a (o intercepto de y) como em b (a inclinação da reta). Se o valor de $b$ for diferente entre as áreas, $o$ maior valor de $b$ apresentará maior incremento de y por incremento de x. Se a inclinação não diferir, mas a constante a for diferente entre áreas, aquelas com maiores valores de a apresentarão valores de y maiores para qualquer valor de x (KOHYAMA; HOTTA, 1990). Foram realizadas as seguintes relações alométricas: altura total x DAP, altura total $\mathrm{x}$ altura de fuste e altura de fuste x DAP.

Foi utilizada a análise de covariância (ANCOVA) para testar a diferença entre as retas (SNEDECOR; COCHRAM, 1967). Comparações múltiplas entre as retas foram feitas pelo teste a posteriori de Scheffé ( $p$ $<0,05$ ) (HUITEMA, 1980; ZAR, 1984).

\section{Resultados}

Considerando a relação entre altura total e DAP, houve diferença significativa entre as inclinações das retas (b) das áreas 1 e 2 se comparadas com a borda, indicando que o aumento de uma unidade na altura provoca um menor aumento no DAP nas plantas da borda (tabela 1 e figura 1). O valor do intercepto em y (a) para as plantas da área 2 foi significativamente maior que o das plantas da área 1. Neste caso, para uma mesma altura, o diâmetro das plantas da área 2 será sempre maior que o das plantas da área 1 . O valor de $\mathrm{r}^{2}$ foi maior para a área 1 , indicando que ocorre maior semelhança na forma dos indivíduos desta área (tabela 1 e figura 1A).

Tabela 1. Estimativa dos parâmetros das regressões lineares para o diâmetro à altura do peito (DAP) $(\mathrm{cm})$ e a altura total $(\mathrm{AT})(\mathrm{m})(\log \mathrm{DAP}=\mathrm{a}+\mathrm{b} \log \mathrm{AT})$ da comunidade arbórea para três áreas do Parque Ecológico da Klabin. Letras iguais na mesma coluna não diferem significativamente entre si (ANCOVA e Teste de Sheffé, $p<0,05$ ). Área 1: interior de uma área em estádio sucessional mais avançado; Borda: borda da área 1; Área 2: interior de uma área em estádio sucessional menos avançado.

\begin{tabular}{lllllll}
\hline Áreas & $\mathrm{a} \#$ & $\mathrm{~b}$ & $\mathrm{r}^{2}$ & $\mathrm{~N}$ & $\mathrm{~b}$ comum & estimativa de a \\
\hline Área 1 & $-4,600$ & $2,160^{\mathrm{a}}$ & $0,662^{* * *}$ & 150 & 2,062 & $-5,461^{\mathrm{b}}$ \\
Borda & $-0,570$ & $1,205^{\mathrm{b}}$ & $0,440^{* * *}$ & 150 & & \\
Área 2 & $-4,202$ & $2,574^{\mathrm{a}}$ & $0,470^{* * *}$ & 144 & & $-3,144^{\mathrm{a}}$ \\
\hline
\end{tabular}

$\# \mathrm{a}=$ intercepto em $\mathrm{y}, \mathrm{b}=$ inclinação da reta, $\mathrm{r}^{2}=$ coeficiente de determinação, $\mathrm{N}=$ número de casos, ANOVA $* * * \mathrm{p}<$ 0,001 .

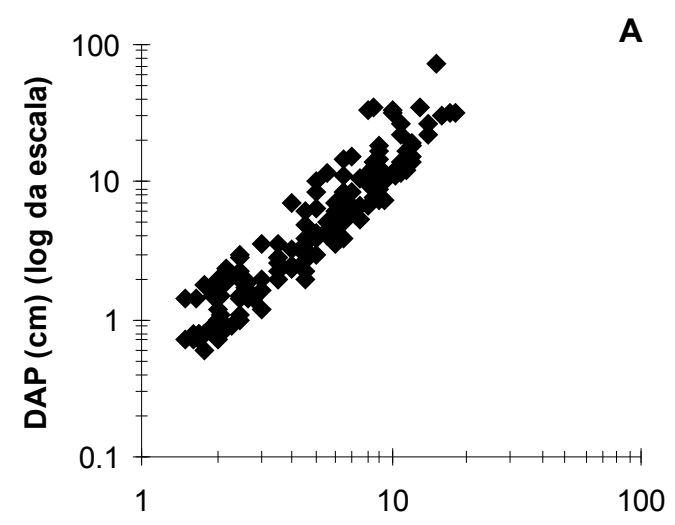

Altura (m) (log da escala)

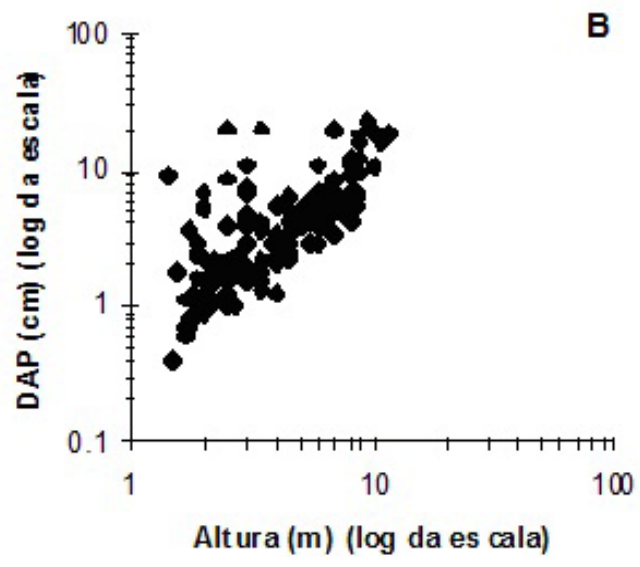




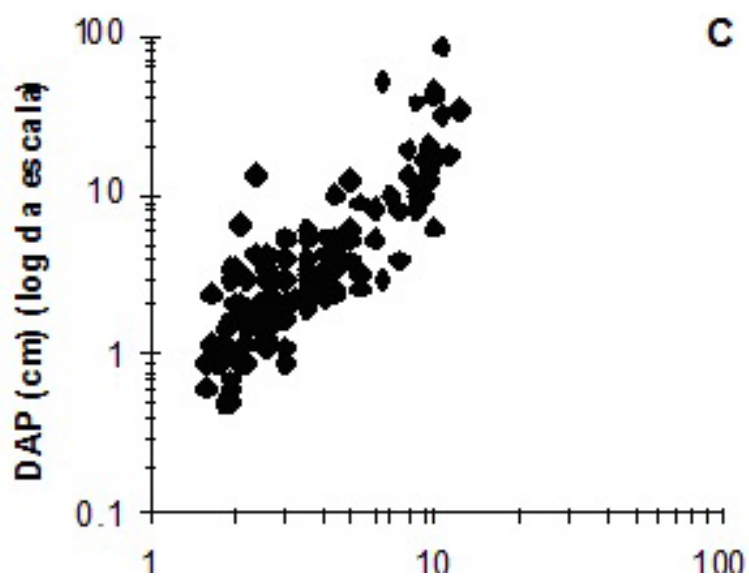

Figura 1. Regressão entre o DAP $(\mathrm{cm})$ e altura total $(\mathrm{m})$ da comunidade arbórea das três áreas $(\log \mathrm{DAP}=\mathrm{a}+\mathrm{b} \log$ AT). A) Área 1; B) Borda; C) Área 2.

Considerando a relação entre altura total e altura de fuste, as inclinações das retas não apresentaram diferenças significativas, entretanto os valores do intercepto de y para as plantas da área 1 e borda foram significativamente maiores que o das plantas da área 2. Isto indica que para uma mesma altura total, os indivíduos da área 2 terão sempre uma

altura de fuste menor que os indivíduos das outras áreas (tabela 2 e figura 2).

Os valores de $\mathrm{r}^{2}$ foram maiores na área 1 e na borda, neste caso, ocorre maior convergência nas formas dos indivíduos destas áreas, quando são consideradas a altura total e a altura de fuste (tabela 2 e figuras $2 \mathrm{~A}$ e $2 \mathrm{~B})$.

Tabela 2. Estimativa dos parâmetros das regressões lineares para a altura do fuste (AF) (m) e a altura total (AT) (m) $(\log \mathrm{AF}=\mathrm{a}+\mathrm{b} \log \mathrm{AT})$ da comunidade arbórea para três áreas do Parque Ecológico da Klabin. Letras iguais na mesma coluna não diferem significativamente entre si (ANCOVA e Teste de Sheffé, $\mathrm{p}<0,05$ ). Área 1: interior de uma área em estádio sucessional mais avançado; Borda: borda da área 1; Área 2: interior de uma área em estádio sucessional menos avançado.

\begin{tabular}{ccccccc}
\hline Áreas & $\mathrm{a} \#$ & $\mathrm{~b}$ & $\mathrm{r} 2$ & $\mathrm{~N}$ & $\mathrm{~b}$ comum & estimativa de a \\
\hline Área 1 & $-0,126$ & $0,624^{\mathrm{a}}$ & $0,673^{* * *}$ & 102 & 0,632 & $-0,183^{\mathrm{a}}$ \\
Borda & $-0,486$ & $0,680^{\mathrm{a}}$ & $0,732^{* * *}$ & 96 & & $-0,272^{\mathrm{a}}$ \\
Área 2 & $-0,813$ & $0,588^{\mathrm{a}}$ & $0,459^{* * *}$ & 107 & & $-1,028^{\mathrm{b}}$ \\
\hline
\end{tabular}

\# $\mathrm{a}=$ intercepto em $\mathrm{y}, \mathrm{b}=$ inclinação da reta, $\mathrm{r}^{2}=$ coeficiente de determinação, $\mathrm{N}=$ número de casos, ANOVA *** $\mathrm{p}<$ 0,001 .
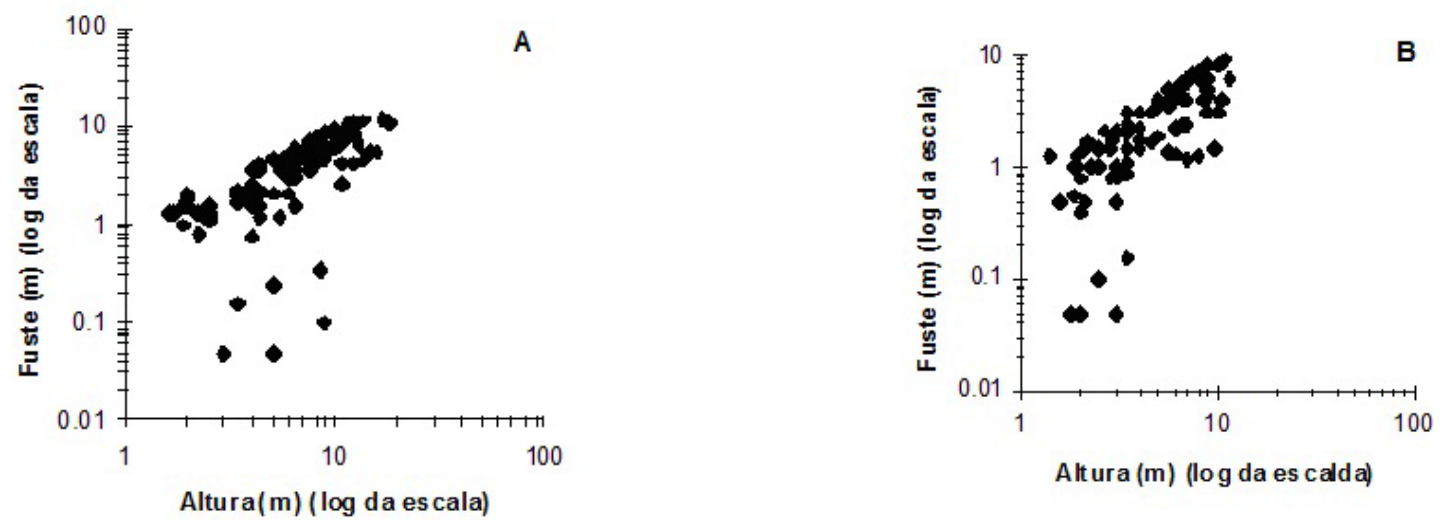


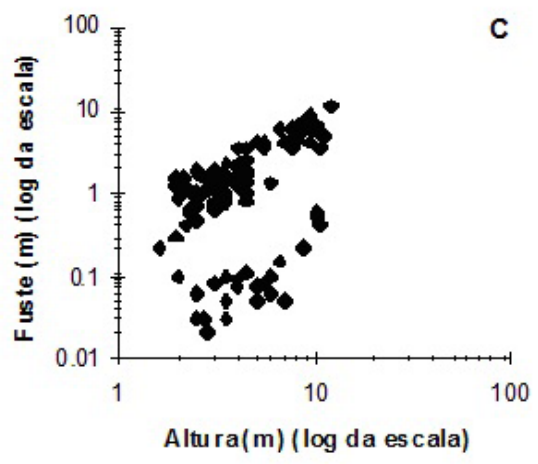

Figura 2. Regressão entre a altura de fuste $(\mathrm{m})$ e altura total $(\mathrm{m})$ da comunidade arbórea das três áreas $(\log \mathrm{AF}=\mathrm{a}+$ b $\log$ AT). A) Área 1; B) Borda; C) Área 2.

Quanto a relação entre altura de fuste e DAP, a inclinação da reta foi maior para a área 1 , indicando que um mesmo aumento na altura de fuste provoca nas plantas desta área um maior aumento no diâmetro (tabela 3 e figura 3). Ou seja, as plantas da área 1 têm maior aumento no DAP por aumento da altura de fuste. $\mathrm{O}$ valor do intercepto em y foi maior para a área 2, comparando-se com a borda. Neste caso, para uma mesma altura de fuste, o diâmetro das plantas da área 2 será sempre maior que o das plantas da borda. Entretanto, o valor de $\mathrm{r}^{2}$ não foi significativo para a área 2 , indicando que nesta área ocorre grandes variações na forma dos indivíduos, quando são considerados a altura de fuste e o DAP (tabela 3 e figura 3C).

Tabela 3. Estimativa dos parâmetros das regressões lineares para o diâmetro à altura do peito (DAP) (cm) e a altura do fuste $(\mathrm{AF})(\mathrm{m})(\log \mathrm{DAP}=\mathrm{a}+\mathrm{b} \log \mathrm{AF})$ da comunidade arbórea para três áreas do Parque Ecológico da Klabin. Letras iguais na mesma coluna não diferem significativamente entre si (ANCOVA e Teste de Sheffé, $\mathrm{p}<0,05$ ). Área 1: interior de uma área em estádio sucessional mais avançado; Borda: borda da área 1; Área 2: interior de uma área em estádio sucessional menos avançado.

\begin{tabular}{llccccc}
\hline Áreas & $\mathrm{a} \#$ & $\mathrm{~b}$ & $\mathrm{r} 2$ & $\mathrm{~N}$ & $\mathrm{~b}$ comum & estimativa de a \\
\hline Área 1 & 2,365 & $1,914^{\mathrm{a}}$ & $0,284^{* * *}$ & 102 & 1,315 & \\
Borda & 2,977 & $0,919^{\mathrm{b}}$ & $0,206^{* * *}$ & 96 & & $3,152^{\mathrm{b}}$ \\
Área 2 & 6,563 & $0,773^{\mathrm{b}}$ & 0,024 & 107 & & $6,403^{\mathrm{a}}$ \\
\hline
\end{tabular}

\# $\mathrm{a}=$ intercepto em $\mathrm{y}, \mathrm{b}=$ inclinação da reta, $\mathrm{r}^{2}=$ coeficiente de determinação, $\mathrm{N}=$ número de casos, ANOVA *** $\mathrm{p}<$
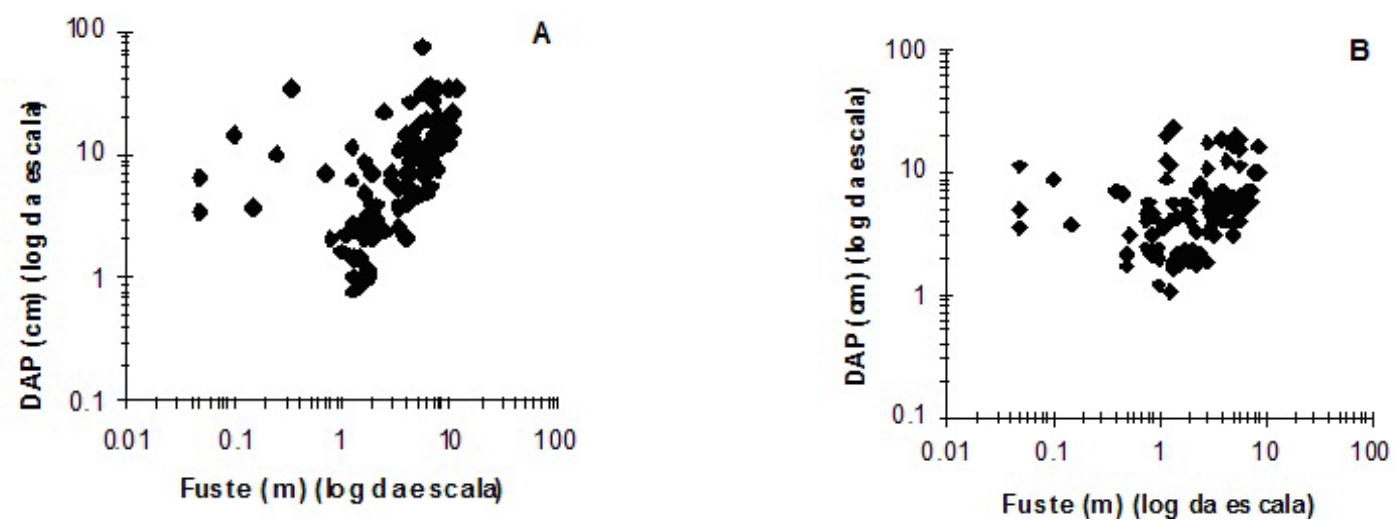


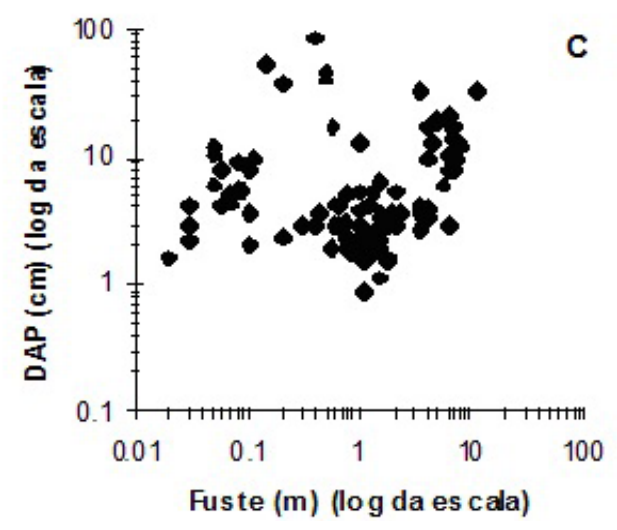

Figura 3. Regressão entre o DAP $(\mathrm{cm})$ e a altura de fuste $(\mathrm{m})$ da comunidade arbórea das três áreas $(\log \mathrm{DAP}=\mathrm{a}+$ b $\log (\mathrm{AF})$. A) Área 1; B) Borda; C) Área 2.

\section{Discussão}

Considerando as relações entre o diâmetro e a altura para comparar a forma dos indivíduos arbóreos das áreas estudadas, observou-se que, para as áreas 1 e 2, os indivíduos apresentaram maior investimento em diâmetro em relação à altura do que os da borda. Isto sugere maior eficiência desses indivíduos em alocar biomassa para o crescimento em espessura, possivelmente devido à maior abundância de espécies de subosque (tolerantes à sombra) nessas áreas, que sobrecarregam o caule por apresentar maior expansão de área fotossinteticamente ativa (maior copa) no microambiente menos iluminado (YAMADA; YAMAKURA; LEE, 2000).

$\mathrm{Na}$ borda ocorre menor incremento em diâmetro, possivelmente devido à colonização desta área por espécies pioneiras que alocam mais biomassa para o crescimento em altura (FONTES, 1999). As espécies pioneiras caracterizam-se por exibir rápido alongamento de um broto monopodial, arquitetura de ramificação simples e baixa densidade de madeira (FONTES, 1999; HUBBELL; FOSTER, 1986). Essas espécies dependem de clareiras para germinação/estabelecimento e apresentam altas taxas de mortalidade em ambientes com baixa disponibilidade de luz (CLARK; CLARK, 1992; SWAINE; WHITMORE, 1988). Segundo Yamada, Yamakura e Lee (2000), espécies que apresentam caules delgados e copas menores parecem estar mais bem ajustados a ambientes onde há maior luminosidade (KAPOS; GANADE; MATSUI, 1993; KAPOS et al., 1997) e, consequentemente, com maiores oportunidades para o crescimento em altura.

$\mathrm{O}$ valor do intercepto em $\mathrm{y}$, considerando ainda as relações entre o diâmetro e a altura, foi significativamente maior para as plantas da área 2 (estádio de sucessão menos avançado) quando comparadas com as da área 1, indicando que para uma mesma altura o diâmetro será sempre maior para as plantas da área 2. Diferente do que foi observado, esperava-se encontrar este resultado pra a área 1, que se apresenta em estádio sucessional mais avançado e possui subosque mais desenvolvido, com espécies tolerantes à sombra (GUARIGUATA et al., 1997; GUARIGUATA; OSTERTAG, 2001), e que são mais eficientes em alocar biomassa para o crescimento em espessura (AIBA; KOHYAMA, 1996). Entretanto, isso pode ser explicado pela alta abundância de algumas espécies da família Myrtaceae típicas da Floresta Ombrófila Mista da região, que foram observadas na área 2. Essas espécies, de baixo porte, apresentaram várias ramificações do caule próximas ao solo (observação pessoal), o que possivelmente contribuiu para o maior intercepto em y observado.

Considerando as relações entre altura da planta e altura do fuste, as plantas da área 2 apresentaram, 
para a mesma altura, menor altura de fuste do que as plantas da área 1 e da borda. Levando em conta as características das espécies que colonizam a borda (preferencialmente pioneiras) é esperado um maior investimento em fuste se comparado com as espécies do interior dos fragmentos. No entanto, essa diferença somente ocorreu quando foi comparado a borda com a área 2. O maior investimento na altura do fuste apresentado pelas plantas da área 1 quando comparadas às da área 2 também pode ser devido a presença das espécies da família Myrtaceae na área 2, que apresentaram altura do fuste muito reduzidas. O menor coeficiente de determinação apresentado pelas plantas da área 2, indicando menor uniformidade na forma dos indivíduos desta área, confirma essa possibilidade. É possível, também, que o fato de não ter havido diferença significativa entre os valores do intercepto em y para a área $1 \mathrm{e}$ a borda, seja em decorrência da maior densidade da vegetação na área 1 , o que aumentou a competição por luz entre os indivíduos. Desta forma, os galhos mais altos seriam favorecidos em detrimento aos mais baixos e os indivíduos desta área estariam alocando mais biomassa para o crescimento em altura, resultando em indivíduos com fuste mais alto e arquitetura semelhante à dos indivíduos da borda. King (1990b) sugere que a competição por luz é o fator primário responsável pela evolução e manutenção da forma de indivíduos arbóreos. Em uma floresta, as plantas competem por luz e quem vence ou perde é determinado em grande parte pela forma da planta (O’BRIEN et al., 1995).

O valor do coeficiente de determinação foi maior para a borda, sugerindo que os indivíduos desta área apresentam maiores semelhanças arquiteturais do que os das outras áreas. A maior heterogeneidade de formas apresentada pelos indivíduos das áreas 1 e 2, provavelmente se deve à colonização por espécies de subosque que se estabelecem mais tardiamente na sucessão florestal (tolerantes à sombra), convivendo com espécies de dossel e emergentes (GUARIGUATA; OSTERTAG, 2001; GUTIÉRREZ et al., 2008). Guariguata e Ostertag
(2001), em uma revisão sobre os principais fatores bióticos e abióticos que influenciam os padrões de sucessão florestal secundária em florestas úmidas neotropicais, ressaltam que a primeira década após o abandono da terra é caracterizada pela presença de gramíneas e arbustos, que posteriormente são sombreados por espécies pioneiras de ciclo de vida curto. Após este período, o dossel é dominado por espécies pioneiras de ciclo de vida longo, mais altas, e que também demandam luminosidade pra se estabelecer. Eventualmente, o dossel desse segundo estádio pode ser substituído por espécies tolerantes à sombra, características de estágios avançados da sucessão florestal, que geralmente germinam e se estabelecem no início da sucessão florestal (GUARIGUATA et al., 1997).

Os indivíduos da área 1 sempre terão, para um mesmo aumento na altura de fuste, maior aumento em diâmetro em comparação aos indivíduos da área 2 e da borda. Esses resultados indicam que para a área que se apresenta em estádio sucessional mais avançado, o aumento do fuste é acompanhado de uma maior expansão da copa, importante para melhor exploração do microambiente menos iluminado do subosque desta área, havendo, portanto, maior necessidade de investir no crescimento em diâmetro. Indivíduos de espécies de subosque apresentam maior expansão do sistema de ramificação lateral e maior alocação de biomassa para a produção de folhas, desta forma devem acumular uma quantidade crescente de biomassa para sustentação (YAMADA; YAMAKURA; LEE, 2000). A semelhança entre a área 2 e borda provavelmente se deve ao fato de ambas as áreas apresentarem maior abundância de espécies pioneiras. Guariguata e Ostertag (2001) propõem uma sequência de eventos que ocorrem durante a sucessão florestal secundária em florestas úmidas neotropicais, até o estádio avançado de sucessão, que apresenta condições semelhantes à da floresta madura. Segundo os autores, entre 20 e 100 anos após o abandono da terra (estágio ocupado pela área 2), o dossel é dominado por espécies pioneiras de ciclo de vida longo. Muitas características e 
processos florestais desta fase da sucessão já se assemelham aos da floresta madura, entretanto os autores destacam que a composição de espécies é o último aspecto a se tornar semelhante à floresta madura.

O crescimento em altura e a expansão da copa refletem estratégias de crescimento em resposta a diferentes condições de luminosidade (AIBA; KOHYAMA, 1996; KOHYAMA, 1987; KOHYAMA; HOTTA, 1990). Essas estratégias de crescimento têm sido interpretadas em relação ao gradiente sucessional, entretanto é provável que essas diferenças também ocorram entre espécies de dossel e subosque, quando se considera o gradiente vertical da floresta (KING, 1990a).

King (1990a) constatou, em uma floresta panamenha, que espécies arbóreas de subosque tendem a ter copas maiores do que indivíduos jovens de espécies de dossel de altura semelhante. Esses padrões foram interpretados, respectivamente, como adaptação para interceptação de luz (em ambiente sombreado) e crescimento em altura como resposta à competição por luz que, segundo King (1996), aumenta a densidade de radiação na superfície da copa (FONTES, 1999).

\section{Conclusão}

Os resultados obtidos estão de acordo com as afirmações de que as modificações das condições ambientais provocadas pelo processo sucessional são muito importantes para explicar a forma dos indivíduos que se estabelecem em diferentes áreas, em razão de melhor explorar os recursos do ambiente. Na área 1, em estádio sucessional mais avançado, ocorre a colonização por espécies tolerantes à sombra, que investem maior biomassa em volume de copa e, por conseqüência, em diâmetro, diferente daquelas dependentes de luz que ocorrem na área 2 e na borda. A área 2, em função do estádio sucessional menos avançado e, consequentemente, da composição de espécies arbóreas desde estádio (p. ex. espécies pioneiras) com indivíduos mais eficientes em alocar biomassa para crescimento em altura, se apresentou mais semelhante à borda, que, devido à improbabilidade de mudanças microambientais principalmente em função da alta luminosidade lateral, não apresenta grandes avanços no processo de sucessão florestal.

A diferença de estádio sucessional com as respectivas espécies arbóreas adaptadas às condições de cada estádio, é a principal causa das variações na arquitetura dos indivíduos da comunidade arbórea das áreas estudadas da Floresta Ombrófila Mista do Parque Ecológico da Klabin, Telêmaco Borba/PR/ Brasil.

\section{Agradecimentos}

À CAPES e ao Programa de Pós Graduação em Ciências Biológicas da Universidade Estadual de Londrina pelo apoio financeiro. À empresa KLABIN S.A. pela acolhida e por permitir a realização dos trabalhos em campo.

\section{Referências}

AIBA, S.; KOHYAMA, T. Tree species stratification in relation to allometry and demography in a warmtemperate rain forest. Journal of Ecology, Oxford, v. 84, n. 2, p. 207-218, abr. 1996.

ALVES, L. F.; METZGER, J. P. A regeneração florestal em áreas de floresta secundaria na Reserva Florestal do Morro Grande, Cotia, SP. Biota Neotropica, Campinas, v. 6, n. 2, maio/ago. 2006.

ALVES, L. F.; SANTOS, F. A. M. Tree allometry and crown shape of four tree species in atlantic rain forest, south-east Brazil. Journal of Tropical Ecology, Cambridge, v. 18, p. 245-260, 2002.

ARCHIBALD, S.; BOND, W. J. Growing tall vs growing wide: tree architecture and allometry of Acacia karroo in forest, savanna, and arid environments. Oikos, Buenos Aires, v. 102, p. 3-14, 2003.

AZEVEDO, T. I. N.; SEKIAMA, M. L.; VIEIRA, A. O. S.; BENNEMANN, S. T. Descrição física da micro bacia do Ribeirão Varanal e caracterização dos trechos. In: BENNEMANN, S. T.; SHIBATTA, O. A.; VIEIRA, A. O. (Org.). A flora e a fauna do Ribeirão Varanal: um 
estudo da biodiversidade no Paraná. Londrina: EDUEL, 2008. p. 7-15.

BARBOSA, C. E. A.; BENATO, T.; CAVALHEIRO, A. L.; TOREZAN, J. M. D. Diversity of regenerating plants in reforestations with Araucaria angustifolia (Bertol.) O. Kuntze of 12, 22, 35, and 43 years of age in Parana state, Brazil. Restoration Ecology, Malden, v. 17, n. 1, p. 6067, 2007.

BENÍTEZ-MALVIDO, J. Impact of forest fragmentation on seedling abundance in a tropical rain forest. Conservation Biology, Boston, v. 12, p. 380-389, 1998.

BERTANI, D. F. Ecologia de populações de Psychotria suterella Müll. Arg. (Rubiaceae) em uma paisagem fragmentada de mata atlântica. 2006. Tese (Doutorado em Biologia Vegetal) - Universidade Estadual de Campinas, Campinas.

BOND, W. J.; HONIG, M.; MAZE, K. E. Seed size and seedling emergence: an allometric relationship and some ecological implications. Oecologia, Berlin, v. 120, p. 132-136, 1999.

CLARK, D. A.; CLARK, D. B. Life history diversity of canopy and emergent trees in a neotropical rain forest. Ecological Monographs, Lawrence, v. 63, n. 3, p. 315344, 1992.

FÁVARO, F. de L.; ANJOS, L. dos; LOPES, E. V.; MENDONÇA, L. B.; VOLPATO, G. H. Efeito do gradiente altitudinal/latitudinal sobre espécies de aves florestais da família Furnariidae na Bacia do Rio Tibagi - PR/Brasil. Revista Brasileira de Zoologia, v.23, p. 261266, 2006.

FONTES, M. A. L. Padrões alométricos em espécies arbóreas pioneiras tropicais. Scientia Forestalis, Piracicaba, v. 55, p. 79-87, jun. 1999.

GUARIGUATA, M. R.; CHAZDON, R. L.; DENSLOW, J. S.; DUPUY, J. M.; ANDERSON, L. Structure and floristics of secondary and old-growth forest stands in lowland Costa Rica. Plant Ecology, Dordrecht, v. 132, p. 107-120, 1997.

GUARIGUATA, M. R.; OSTERTAG, R. Neotropical secondary succession: changes in structural and functional characteristics. Forest Ecology and Management, Amsterdam, v. 148, p. 185-206, 2001.

GUTIÉRREZ, A. G.; ARAVENA, J. C.; CARRASCOFARÍAS, N. V.; CHRISTIE, D. A.; FUENTES, M.; J. J. ARMESTO. Gap-phase dynamics and coexistence of a long-lived pioneer and shade-tolerant tree species in the canopy of an old-growth coastal temperate rain forest of Chiloé Island, Chile. Journal of Biogeography, Oxford, v. 35, p. 1674-1687, 2008.
HUBBELL, S. P.; FOSTER, R. B. Canopy gaps and the dynamics of a neotropical forest. In: CRAWLEY, M. J. (Ed.). Plant Ecology. Oxford: Blackwell Scientific Publications, 1986. p. 77-96.

HUITEMA, B. E. The analysis of covariance and alternatives. New York: Wiley, 1980.

KAPOS, V.; GANADE, G.; MATSUI, E.; R. L. VICTORIA d13C as an indicator of edge effects in tropical rainforest reserves. Journal of Ecology, Oxford, v. 81, p. 425-432, 1993.

KAPOS, V.; WANDELLI, E.; CAMARGO, J. L.; GANADE, G. Edge-related changes in environment and plant responses due to forest fragmentation in central amazonia. In: LAURANCE, W. F.; BIERREGAARD, R. O. (Ed.). Tropical forest remnants: ecology, management and conservation of fragmented communities. Illinois: University of Chicago Press, 1997. p. 33-44.

KING, D. A. Allometry of saplings and understorey trees of a Panamanian forest. Functional Ecology, Oxford, v. 4, p. 27-32, 1990a.

Alometry and life history of tropical trees. Journal of Tropical Ecology, Cambridge, v. 12, p. 25-44, 1996.

The adaptive significance of tree height. American Naturalist, Chicago, v. 135, p. 809-828, 1990 b.

KOHYAMA, T. Significance of architecture and allometry in saplings. Functional Ecology, Oxford, v. 1, p. 399-404, 1987.

KOHYAMA, T.; HOTTA, M. Significance of allometry in tropical saplings. Functional Ecology, Oxford, v. 4, p. 515- 521, 1990.

LAURANCE, W. F.; FERREIRA, L. V.; RANKIN-DEMERONA, J. M.; LAURANCE, S. G.; HUTCHINGS, R. W.; LOVEJOY, T. E Effects of forest fragmentation on recruitment patterns in Amazonian tree communities. Conservation Biology, Boston, v. 12, p. 460-464, abr. 1998.

MENDONÇA, F. A.; DANNI-OLIVEIRA, I. M. Dinâmica atmosférica e tipos climáticos predominantes da bacia do rio Tibagi. In: MEDRI, M. E.; BIANCHINI, E.; SHIBATTA, O. A.; PIMENTA, J. A. (Ed.). A bacia do rio Tibagi. Londrina: EDUEL, 2002. p. 63-66.

NASCIMENTO, H. E. M.; LAURANCE, W. F. Efeitos de área e de borda sobre a estrutura florestal em fragmentos de floresta de terra-firme após 13-17 anos de isolamento. Acta Amazonica, Manaus, v. 36, n. 2, p. 183-192, 2006.

NIKLAS, K. J. Plant allometry: the scaling of form and process. Chicago: The University of Chicago Press, 1994. 
O'BRIEN, S. T.; HUBBELL, S. P.; SPIRO, P.; RICHARD, C.; FOSTER, R. B. Diameter, height, crown and age relationships in eight neotropical tree species. Ecology, Oxford, v. 76, p. 1926-1939, 1995.

PORTELA, R. C.; SANTOS, F. A. M. Alometria de plântulas e jovens de espécies arbóreas: copa $\mathrm{x}$ altura. Biota Neotrópica, Campinas, v. 3, n. 2, p. 1-5, 2003.

SILVA, F. M. R.; THEY, N. H.; TROIAN, V. R. R.; KINDLE, A. Densidade populacional e relações alométricas de Psychotria leiocarpa Cham. \& Schltdt. (Rubiaceae) em paisagem fragmentada no Morro Santana, Porto Alegre, RS. Revista Brasileira de Biociências, Porto Alegre, v. 5, n. 1, p. 486-488, jul. 2007.

SNEDECOR, G. W.; COCHRAN, W. G. Statistical methods. 6th. ed. Ames: Iowa State University Press, 1967.

SOKAL, R. R.; ROHLF, F. J. Biometry. 2. ed. San Francisco: Freeman, 1981.

SPOSITO, T. C.; SANTOS, F. A. M. Scaling of stem and crown in eight Cecropia (Cecropiaceae) species of Brazil. American Journal of Botany, Columbus, v. 88, p. 939-949, 2001.

SWAINE, M. D.; WHITMORE, T. C. On the definition of ecological species groups in tropical forests. Vegetation, Knivsta, v. 75, p. 81-86, 1988.

WALLER, D. M. The dynamics of growth and form. In: CRAWLEY, M. J. (Ed.). Plant ecology. Oxford: Blackwell Scientific Publications, 1986. p. 291-320.

WILLIAMS-LINERA, G. Vegetation structure and environmental conditions of forest edges in Panama. Journal of Ecology, Oxford, v. 78, p. 356-373, 1990.

YAMADA, T.; YAMAKURA, T.; LEE, H. S. Architectural and allometric differences are related to microhabitat preferences. Functional Ecology, Oxford, v. 14, p. 731-737, 2000.

ZAR, J. H. Biostatistical analysis. 2. ed. New Jersey: Prentice Hall, 1984.

Recebido em: 18 de março de 2010

Aceito em: 10 de junho de 2010 